\title{
Crosstalk Between Androgen-sensitive and Androgen-insensitive Prostate Cancer Cells
}

\author{
YUTA TAKEZAWA, KOUJI IZUMI, KAZUAKI MACHIOKA, HIROAKI IWAMOTO, \\ RENATO NAITO, TOMOYUKI MAKINO, SUGURU KADOMOTO, ARIUNBOLD NATSAGDORJ, \\ YOSHIFUMI KADONO, EVAN T. KELLER, JIAN ZHANG and ATSUSHI MIZOKAMI \\ Integrative Cancer Therapy and Urology, Division of Cancer Medicine, \\ Graduate School of Medical Sciences, Kanazawa University, Kanazawa, Japan
}

\begin{abstract}
Aim: To investigate how androgen-sensitive LNCaP cells crosstalk with androgen-insensitive DU145 or PC-3 cells. Materials and Methods: The numbers of LNCaP cells were counted when co-cultured with DU145 or PC-3 cells and vise versa. Androgen receptor (AR) activity in LNCaP cells was examined by luciferase reporter assay after transfection with a luciferase reporter driven by PSA promoter in the presence of DU145 or PC-3 cells. Concentration of androgens in the medium was measured by liquid chromatography-mass spectrometry (LC-MS/MS). The ability of migration and invasion of PC-3 and DU145 cells was investigated using a 2-layer chamber, in the presence of LNCaP cells. Results: Co-culture of LNCaP cells with DU145 cells resulted in the conversion of dehydroepiandrosterone (DHEA) to dihydrotestosterone (DHT), which stimulated cell proliferation and PSA promoter activity in LNCaP cells. The increased cell proliferation rate and AR activity, induced in LNCaP cells after DHT treatment, was further enhanced by co-culture with DU145 cells. LNCaP cells also stimulated the proliferation of DU145 and PC-3 cells, via secreting soluble factors. Finally, LNCaP cells promoted migration and invasion of PC-3 cells, in a co-culture system; however inhibited migration and invasion of DU145 cells. Conclusion: Crosstalk between androgen-sensitive PCa cells and androgen-insensitive PCa cells might develop the progression of PCa.
\end{abstract}

Prostate cancer (PCa) is the most common malignancy and the second leading cause of cancer-related death in men in

Correspondence to: Atsushi Mizokami, Department of Integrative Cancer Therapy and Urology, Graduate School of Medical Science, Kanazawa University, 13-1 Takara-machi Kanazawa, Ishikawa 9208640 Japan. Tel: +81 762652393, Fax: +81 762344263, E-mail: mizokami@staff.kanazawa-u.ac.jp

Key Words: Prostate cancer, androgen, androgen-sensitive, androgeninsensitive. the United States (1). Advanced PCa is initially dependent on androgens, and thus, androgen-deprivation therapy (ADT) is the standard of care. However, after an initial response to ADT, PCa eventually becomes androgen-independent and progresses to castration-resistant prostate cancer (CRPC) (2).

Development of advanced PCs to CRPC during ADT is mediated via multiple molecular mechanisms, classified in the following two: adaptation to the low androgen concentration caused by ADT, and clonal selection (3-6). In order to adapt to the low concentration of androgen from adrenal gland after ADT, PCa cells synthesize testosterone from dehydroepiandrosterone (DHEA) in an intracrine fashion $(7,8)$. Especially, PCa-derived stromal cells have been reported to promote the production of androgen, dihydrotestosterone (DHT), from DHEA $(9,10)$. Moreover, PCa cancer often metastasizes to bones, where bone microenvironment also contributes to cancer metastasis and progression (11). Transforming growth factor- $\beta$ (TGF- $\beta 1$ ), which is released by osteoblasts and bone marrow stromal cells, has been shown to increase DHT synthesis in DHEAtreated primary PCa cells and also, to alter the expression of genes encoding androgen metabolism-related enzymes (12, 13). On the basis of this evidence, strong androgen receptor (AR) axis target drugs, abiraterone acetate and enzalutamide, have been developed and their effectiveness against CRPC has been clinically proven, suggesting that intracrine androgen biosynthesis contributed to the development of CRPC $(14,15)$. However, their effects are not long-lasting and new therapeutic approaches are required for the treatment of advanced CRPC (16).

Tumor stroma in PCa harbors various cell types, including fibroblasts, macrophages, and endothelial cells, which also play a crucial role in the progression of PCa. Phenotypically modulated fibroblasts, termed myofibroblasts, interact with epithelial and other cell types of the connective tissue and thus, may control tumor invasion and angiogenesis (17). Crosstalk of tumor-associated stromal cells and epithelial cells, mediated through various cytokines and chemokines, 
plays an active role in the progression, androgen-independent conversion, and distal metastasis of PCa (18). The paracrine interplay between cancer-associated fibroblasts (CAFs) and cancer cells also leads to an epithelial-mesenchymal transition (EMT)-driven gain of cancer stem cell properties associated with aggressiveness and metastatic spread (19). Cytokines and growth factors secreted by cells in the bone marrow attract and support cancer cells, thus promoting growth development and metastasis of the tumor $(20,21)$. More specifically, growth rate of PCa cells has been shown to accelerate when they enter the bone environment, suggesting that factors present in bone stimulate tumor cell proliferation (22).

Herein, we hypothesized that interplay between androgensensitive and androgen-insensitive PCa cells plays a critical role in the development of advanced PCa to CRPC, similarly to the previously described crosstalk of androgen-sensitive PCa cells with PCa-derived stromal cells (10). Therefore, androgen-sensitive and androgen-insensitive PCa cells were co-cultured, and cell proliferation and AR activity were investigated in androgen-sensitive LNCaP cells. Additionally, androgen synthesis, as well as migration and invasion of androgen-insensitive PCa cells were explored.

\section{Materials and Methods}

Cell culture. LNCaP, PC-3, and DU145 cell lines were purchased from American Type Culture Collection (ATCC, Manassas, VA, USA). LNCaP and DU145 cells were cultured in Dulbecco's Modified Eagle's Medium (DMEM; Sigma-Aldrich, St. Louis, MO, USA) including 5\% fetal bovine serum (FBS; Sigma-Aldrich, St. Louis, MO, USA), PC-3 cells were cultured in RPMI medium (Sigma-Aldrich, St. Louis, MO, USA) including 5\% FBS.

Co-culture and cell proliferation. LNCaP cells $\left(5 \times 10^{4}\right)$ were seeded on 12-well plates (2-layer chambers) with DMEM including 5\% charcoal-stripped fetal (CCS) (HyClone Laboratories, Logan, UT, USA). After $24 \mathrm{~h}, \mathrm{DU} 145$ or PC-3 $\left(10 \times 10^{4}\right)$ cells were plated on the upper chamber (1.0 $\mu \mathrm{m}$ pore size 12-well format; Becton Dickinson, Franklin Lakes, NJ, USA) with DMEM containing 5\% CCS for 24 h. Afterwards, cells were treated with DHEA $(0.3,10,30 \mathrm{nM})$ or DHT $(0,0.1$, and $1.0 \mathrm{nM})$ and cultured for 4 days. Medium was replaced once, at day 2 of treatment. $\mathrm{LNCaP}$ cells cultured alone for 4 days served as control. In the same way, $2 \times 10^{4}$ DU145 or PC-3 cells were seeded on 12-well plates with DMEM containing 5\% CCS, for $24 \mathrm{~h}$, and then $5 \times 10^{4} \mathrm{LNCaP}$ cells were plated in cell culture inserts for additional $24 \mathrm{~h}$ and treated with $1 \mathrm{nM}$ DHT or $10 \mu \mathrm{M}$ enzalutamide (ENZ) for 4 days. Medium was replaced once, at day 2 of treatment. To determine cell proliferation, cell inserts were removed and cells in the bottom wells were trypsinized and counted in triplicate using a hemocytometer. The data represent the means \pm SD of three replicates.

Culture with conditioned medium (CM). CM was obtained from subconfluent LNCaP cells cultured in DMEM-5\% CCS medium, for $24 \mathrm{~h}$, in a $75 \mathrm{~cm}^{2}$ flask. DU145 or PC- 3 cells $\left(3 \times 10^{4}\right.$ or $2 \times 10^{4}$, respectively) were cultured with $\mathrm{CM}$ for 4 days, and medium was replaced at day 2. CM was used diluted at ratio of 1:4 or 2:4 with DMEM containing $5 \%$ CCS.

In vitro luciferase assay. To examine $\mathrm{AR}$ activity, luciferase reporter assay was performed as described previously using $0.4 \mu \mathrm{g}$ luciferase reporter plasmid, pGL-5.8PSAp, under the control of 5.8-kb PSA promoter including androgen-response elements (23). Twelve $\mathrm{h}$ after transfection, $5 \times 10^{4} \mathrm{LNCaP}$ cells were co-cultured with $5 \times 10^{4}$ DU145 or PC-3 cells on the same plates for $12 \mathrm{~h}$, followed by incubation with 0,10 , and $100 \mathrm{nM}$ DHEA or $0,0.1$, and $1.0 \mathrm{nM}$ DHT, for $24 \mathrm{~h}$, as previously described (10). Monoculture of transfected LNCaP cells, treated with DHEA or DHT for $24 \mathrm{~h}$, served as control. Cells were then harvested and lysed in luciferase lysis buffer (Promega, Madison, WI, USA).

Quantitative analysis of androgens in the medium by liquid chromatography-mass spectrometry (LC-MS/MS). The concentration of androgens DHEA, T, and DHT, in medium from co-cultures was measured using LC-MS/MS, as previously described (10). Medium was collected before harvesting cells for luciferase assay, it was stored at $-30^{\circ} \mathrm{C}$, and LC-MS/MS was performed by ASUKA Pharmaceutical Medical Co. Ltd (Japan). The limits of quantification of DHEA, T, and DHT in the medium were $4 \mathrm{pg} / \mathrm{ml}, 2 \mathrm{pg} / \mathrm{ml}$, and $2 \mathrm{pg} / \mathrm{ml}$, respectively according to ASUKA.

Western blot analysis. To examine levels of proteins related to EMT, $1 \times 10^{5}$ DU145 or PC- 3 cells were seeded for $24 \mathrm{~h}$. And then, they were co-cultured with $2 \times 10^{5} \mathrm{LNCaP}$ cells cells in RPMI containing $10 \% \mathrm{CCS}$ for $24 \mathrm{~h}$, using 6-well plates and cell culture inserts. Afterwards, protein extraction from PC-3 and DU145 cells was performed as previously described (24). Primary antibodies against E-cadherin (mouse monoclonal, Abcam, Cambridge, UK), Ncadherin (mouse monoclonal, Abcam, Cambridge, UK), SNAIL (mouse monoclonal, Abcam, Cambridge, UK) and vimentin (rabbit monoclonal, Abcam, Cambridge, UK) were used. The secondary antibodies were goat anti-mouse HRP conjugate (1:10,000, Bio-Rad Laboratories, Hercules, CA, USA) or anti-rabbit IgG, HRP-linked antibody (1:1,000, Cell Signaling Technology, Danvers, MA, USA). Protein bands were detected using SuperSignal West Femto Maximum Sensitivity Substrate (ThermoFisher, Waltham, MA, USA), visualized by ChemiDoc XRS (Bio-Rad Laboratories, Hercules, CA, USA), and analyzed using Quantity One v4.6.5 software (Bio-Rad Laboratories, Hercules, CA, USA).

Cell migration and invasion assay. To examine the migratory ability of DU145 and PC- 3 cells, $2 \times 10^{5}$ LNCaP cells were cultured for 24 h, on 24-well plates with RPMI containing $10 \%$ CCS, and then $5 \times 10^{3}$ DU145 or PC-3 cells were plated in cell culture inserts $(8.0 \mu \mathrm{m}$ pore size 24-well format; Life Sciences, One Becton Circle, Durham, USA) for $24 \mathrm{~h}$. Migrated cells were fixed with paraformaldehyde and stained with Crystal Violet (Sigma, St. Louis, MO, USA) for 20-30 min, and cell number was counted. Moreover, for the cell invasion assay, LNCaP cells $\left(2 \times 10^{5}\right)$ were cultured for $12 \mathrm{~h}$, on 24 -well plates using RPMI-10\% CCS. DU145 or PC-3 cells $\left(1 \times 10^{5}\right)$ were then plated in cell culture inserts containing Matrigel barrier (CORNING, Corning, NY, USA), for $24 \mathrm{~h}$. Afterwards, matrigel was removed and invaded cells were fixed by paraformaldehyde, and then, stained with Crystal Violet for 20-30 min. Acetic acid was added for 50-60 min to solubilize Crystal Violet and the optical density (OD) was measured on a microreader at $595 \mathrm{~nm}$. 
A

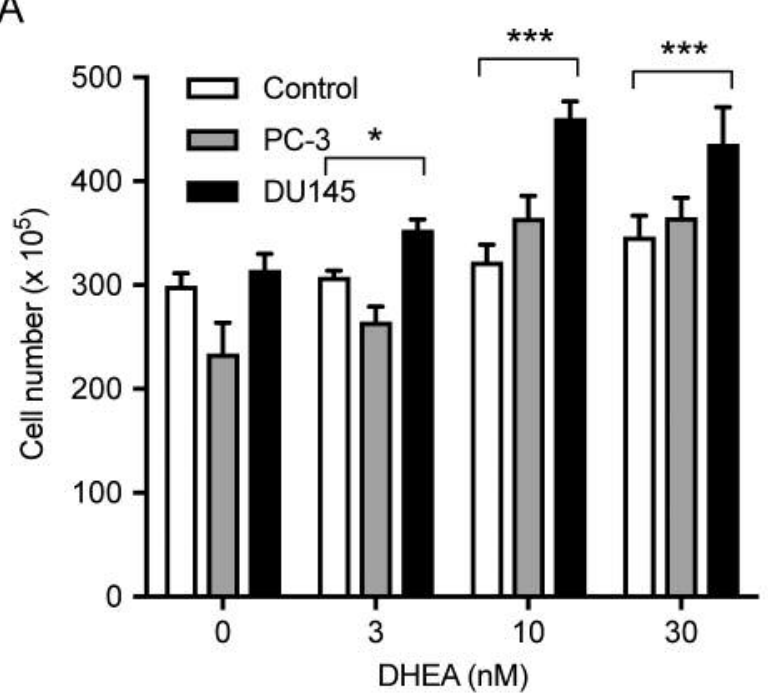

C

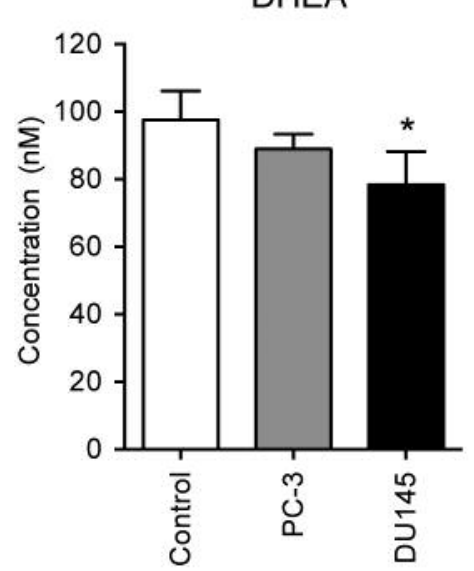

B

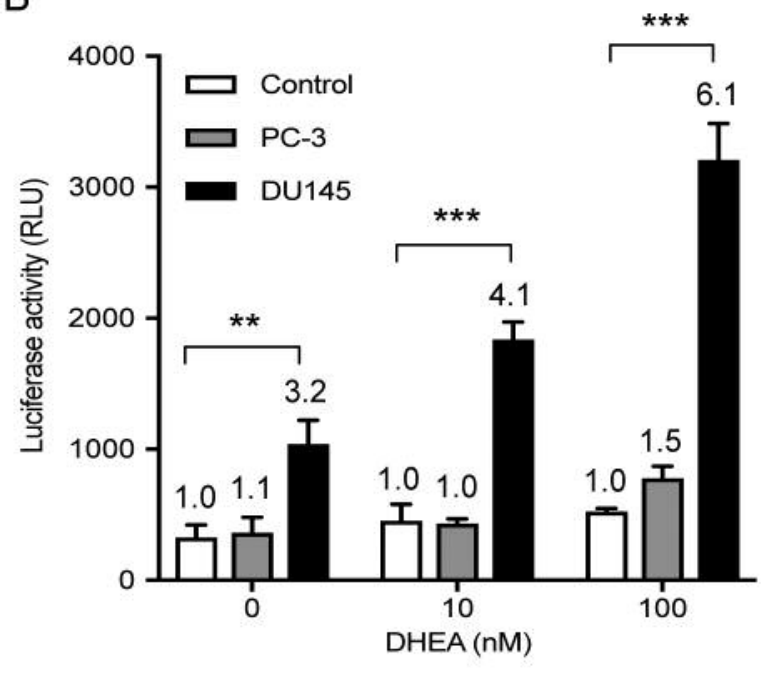

T

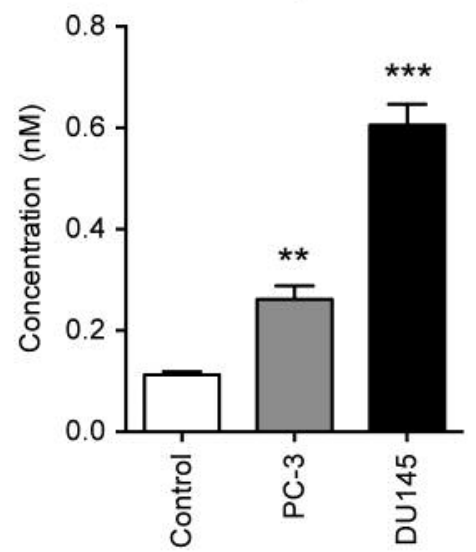

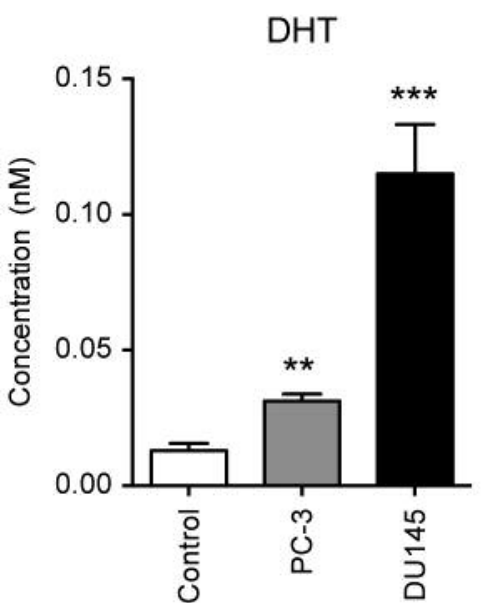

Figure 1. Proliferation and androgen receptor (AR) activity of LNCaP cells co-cultured with PC-3 and DU145 cells in the presence of dehydroepiandrosterone (DHEA). (A) Cell proliferation assay. LNCaP cells were co-cultured with PC-3 or DU145 (no contact) for 24 h and were treated with an indicated concentration of DHEA for 4 days. (control: no co-culture) (B) Luciferase reporter assay. Twelve $h$ after transfection of LNCaP cells with luciferase reporter pGL3PSAp-5.8, LNCaP cells were co-cultured with PC-3 or DU145 cells on the same dish for $12 \mathrm{~h}$, followed by the addition of indicated concentration of DHEA for $24 \mathrm{~h}$. The number above each column represents fold-change compared to the respective control. (control: no co-culture) (C) Quantitative analysis of DHEA, testosterone (T), and dihydrotestosterone (DHT) in medium from DHEA-treated co-cultures by liquid chromatography-mass spectrometry (LC-MS/MS). (control: no co-culture). Asterisks represent statistical significance $\left({ }^{*} p<0.05\right.$, ${ }^{* *} p<0.01$, and $* * * p<0.0001$ between columns linked in a line).

Statistical analysis. Student's $t$-test was used to determine the statistical significance of comparisons between two groups, regarding proliferation, luciferase assays, androgen concentration, and migration/invasion. A $p$-value $<0.05$ was considered statistically significant. These experiments were performed at least twice in order to confirm reproducible results, and we got similar results.

\section{Results}

Androgen-independent DU145 cells enhance biosynthesis of testosterone from DHEA. It has been previously revealed that
PCa-derived stromal cells and androgen-sensitive LNCaP cells could coordinately activate androgen biosynthesis of DHT from DHEA and enhance LNCaP cell proliferation in the presence of DHEA (10). However, androgen-independent PCa cells also co-exist in PCa microenvironment (3). In particular, androgen-independent DU145, but not PC-3 cells, have been confirmed to express type $517 \beta$-hydroxysteroid dehydrogenase (HSD17B5) that converts DHEA to the intermediate in testosterone biosynthesis, androstenediol. HSD17B5 is also expressed by PCa-derived stromal cells 
A

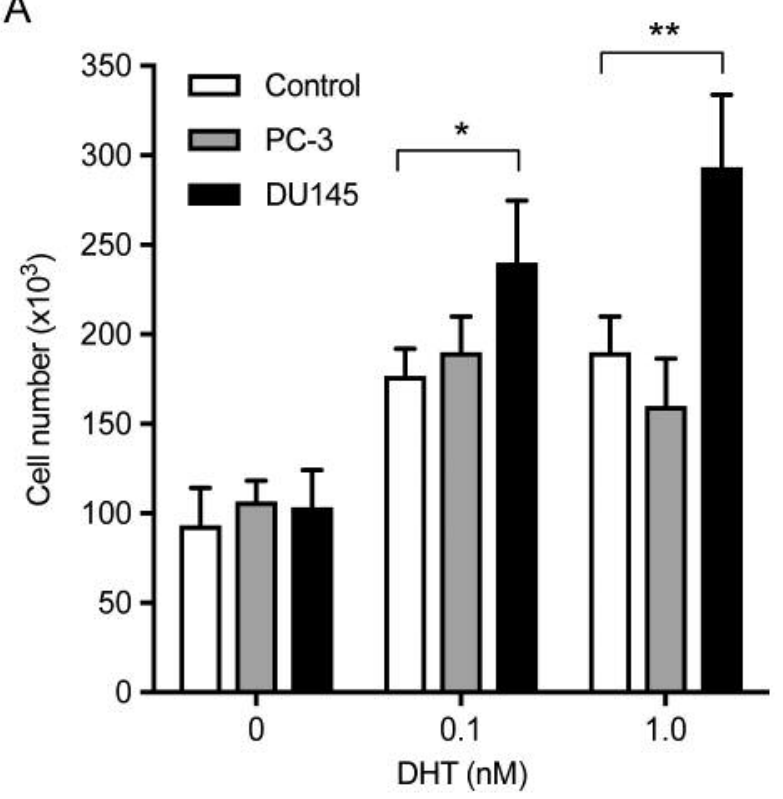

C

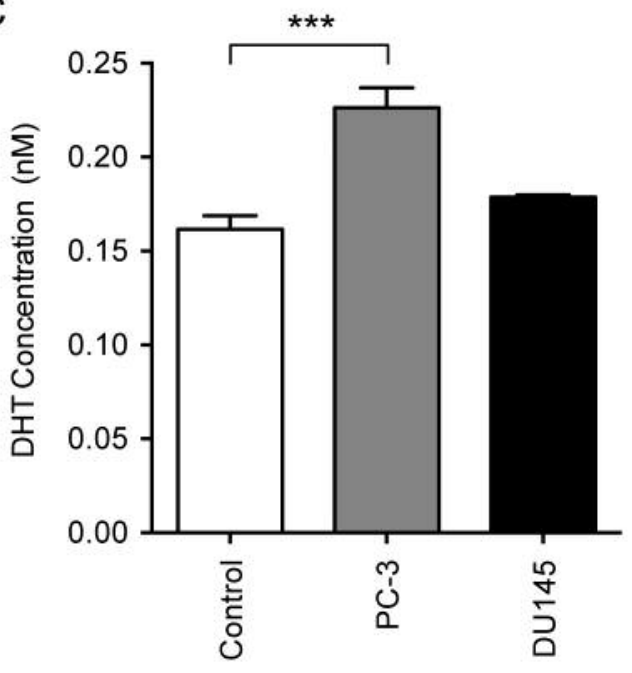

(10). Therefore, based on our previous findings on PCaderived stromal cells, we speculated that DU145 cells might also contribute to androgen biosynthesis and enhance the proliferation of LNCaP cells in the presence of DHEA. When LNCaP cells were treated with DEHA, their proliferation was hardly stimulated, irrespective of the concentration of DHEA. In contrast, when LNCaP cells were co-cultured with DU145, but not with PC-3 cells, their proliferation was stimulated by 1.14 -fold $(p<0.05), 1.43$-fold $(p<0.001)$, and 1.25 -fold $(p<0.001)$ after 3,10 , and $30 \mathrm{nM}$ DHEA treatment, respectively (Figure 1A). Furthermore, AR activity in LNCaP was investigated in the same model. LNCaP cells were transfected with a luciferase reporter (pGL3PSAp-5.8) under the control of 5.8-kb PSA promoter,
B

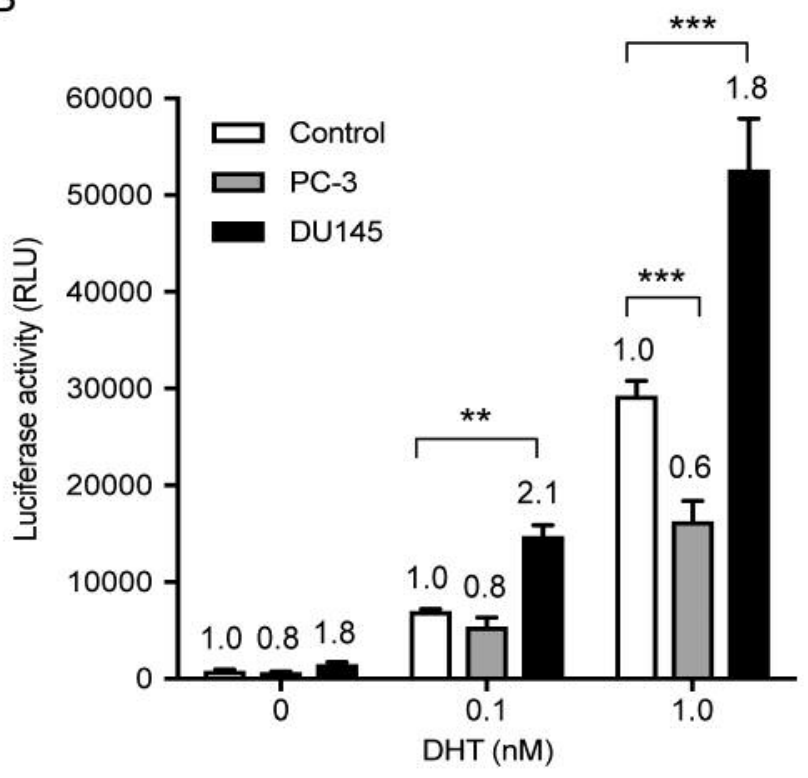

Figure 2. Proliferation and androgen receptor (AR) activity of LNCaP cells after co-culture with PC-3 and DU145 cells. (A) LNCaP cells were co-cultured with PC-3 or DU145 cells and treated with indicated concentration of dihydrotestosterone (DHT) for 4 days (control: no coculture). (B) Luciferase reporter assay. Twelve hours after transfection of LNCaP cells, LNCaP cells were co-cultured with PC-3 or DU145 cells for $12 \mathrm{~h}$, followed by the addition of indicated concentration of DHT for $24 \mathrm{~h}$. The number above each column represents fold-change compared to the respective control (control: no co-culture). (C) Quantitative analysis of DHT concentration in the medium of LNCaP cells co-cultured with PC-3 or DU145 cells, after DHT treatment. Asterisks represent statistical significance $\left({ }^{*} p<0.05,{ }^{*} p<0.01\right.$, and $*^{* *} p<0.0001$ between columns linked in a line).

which is a well-known androgen-responsive promoter (23). DHEA (100 nM) treatment hardly induced AR activity in LNCaP monoculture. In contrast, co-culture of LNCaP cells with DU145 cells induced AR activity by 3.2 -fold $(p<0.001)$ compared to LNCaP monoculture in the absence of DHEA. AR activity by $100 \mathrm{nM}$ DHEA was further increased by 6.1 fold $(p<0.0001)$ in co-culture of LNCaP cells with DU145 cells (Figure 1B). However, DHEA-induced AR activity was not altered in LNCaP cells co-cultured with PC-3 cells.

We explored whether the activation of AR activity in LNCaP cells co-cultured with DU145 cells in the presence of DHEA might be due to induction of DHT synthesis from DHEA. Then, LC-MS/MS was used to estimate the concentration of DHEA, testosterone (T), and DHT in the 
A

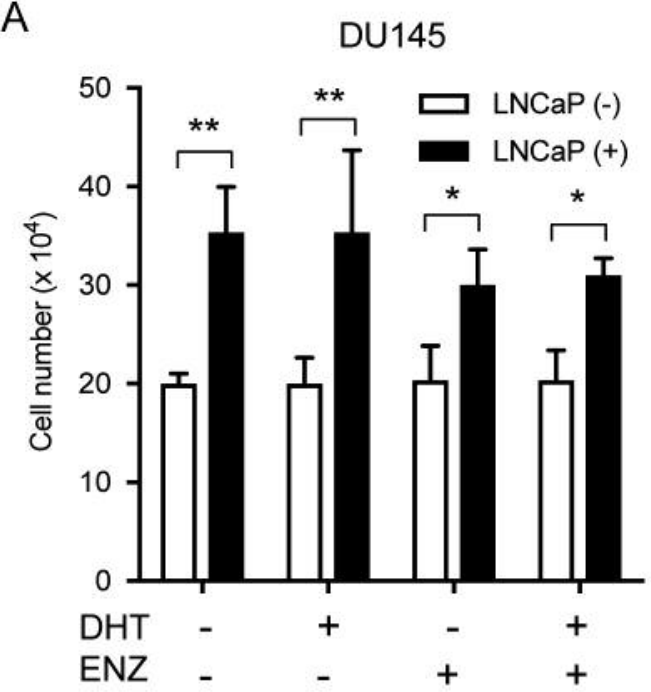

B

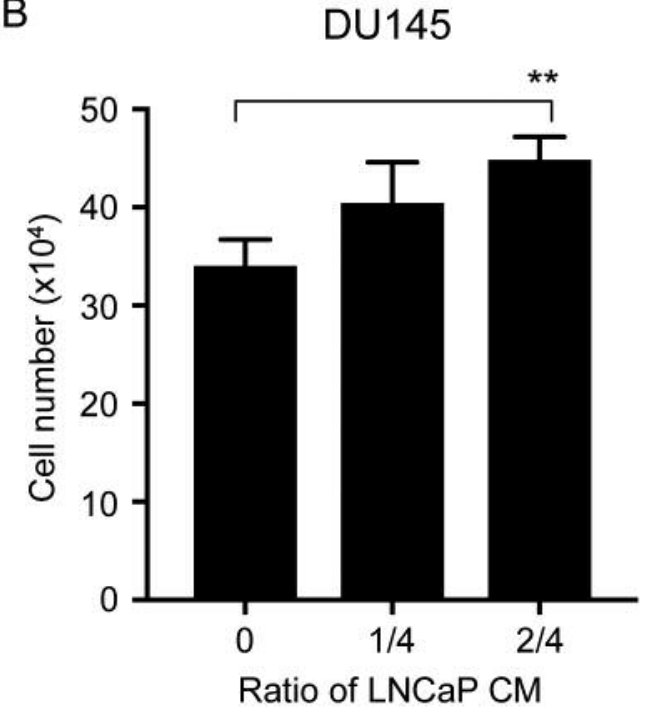

PC-3

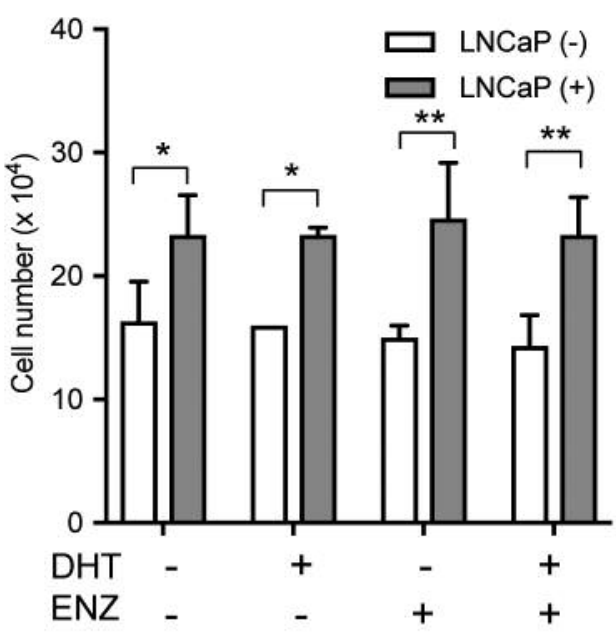

PC-3

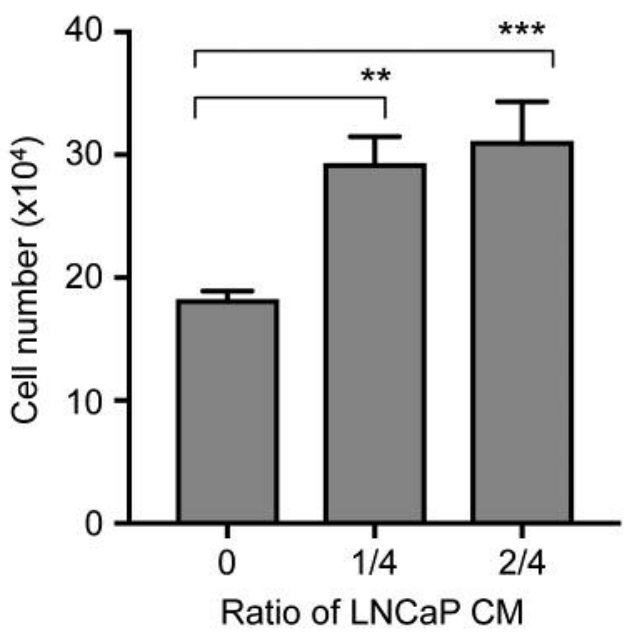

Figure 3. Effect of LNCaP cells on the proliferation of DU145 and PC-3 cells. (A) DU145 or PC-3 cells were co-cultured with LNCaP cells on an two-layer insert (no contact) for $24 \mathrm{~h}$ and treated with $1 \mathrm{nM}$ dihydrotestosterone (DHT) or $10 \mu \mathrm{M}$ enzalutamide (ENZ) for 4 days. (B) DU145 or $P C-3$ cells were co-cultured with or without CM from LNCaP cells and counted after 4 days. Asterisks represent statistical significance $(* p<0.05$, $* * p<0.01$, and $* * * p<0.00010 .01$ compared to white column).

medium from co-cultures of LNCaP cells with PC-3 or DU145 cells, after a 24-h treatment with $100 \mathrm{nM}$ DHEA (Figure 1C). Co-culture with DU145 cells elevated the concentration of T and DHT (5- and 8-fold of control, respectively, both $p<0.0001$ ), while co-culture with PC-3 cells led to a slighter increase of $\mathrm{T}$ and DHT concentration (both 2 -fold, $p<0.01$ ). These results indicated that the increased DHT synthesis by DU145 cells might activate AR and also elevate the proliferation of LNCaP cells in the presence of DHEA.
DU145 cells further enhance AR activity induced by DHT in LNCaP cells. It was further examined whether androgenindependent PCa cells could enhance the proliferation of LNCaP cells in the presence of DHT, in a 2-layer co-culture system (Figure 2A). When LNCaP cells were treated with DHT, the proliferation was stimulated by 0.1 and $1.0 \mathrm{nM}$ DHT. The proliferation of LNCaP cells in the presence of 0.1 and $1.0 \mathrm{nM}$ DHT was further enhanced to 1.36 -fold $(p<0.05)$ and 1.54 -fold, $(p<0.001)$, respectively by co-culture with DU145 cells compared to LNCaP monoculture. However, 

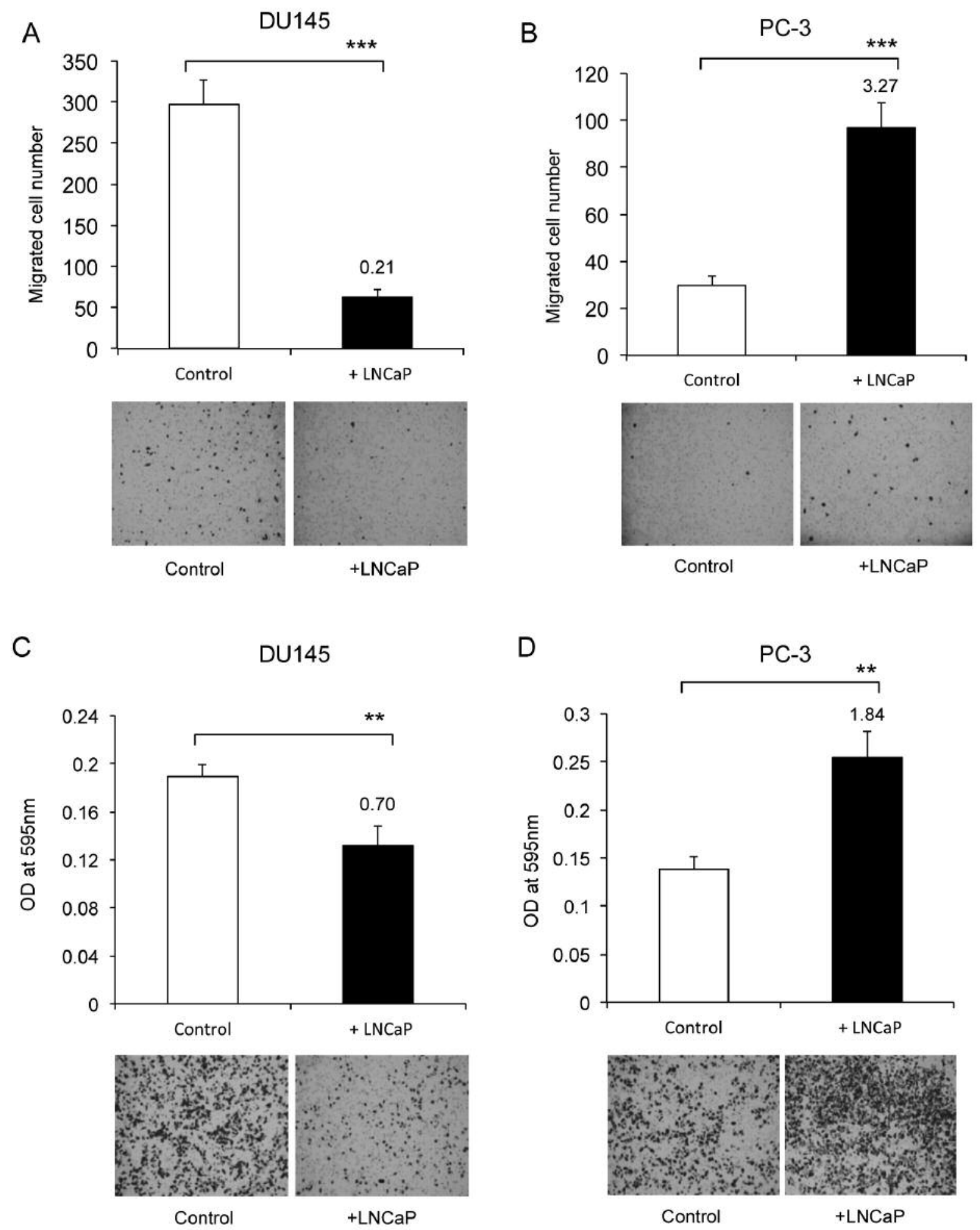

Figure 4. Cell migration and invasion assay after co-culture of LNCaP with DU145 or PC-3 cells. (A, B) Migration of DU145 and PC-3 cells. LNCaP cells were co-cultured with DU145 or PC-3 cells (no-contact) for $24 \mathrm{~h}$. Migrated cells were fixed and counted after staining with Crystal Violet. The numbers above the black columns represent fold change compared to the control (no co-culture). (C, D) Invasion of DU145 and PC-3 cells. LNCaP cells were co-cultured with DU145 or PC-3 cells (no-contact) for $24 \mathrm{~h}$, with the addition of matrigel barrier in cell culture inserts. Invaded cells were fixed and stained with Crystal Violet. The numbers above the black columns represent fold change compared to the control. Asterisks represent statistical significance $(* * p<0.01$ and $* * * p<0.0001$ compared to white column). 
A

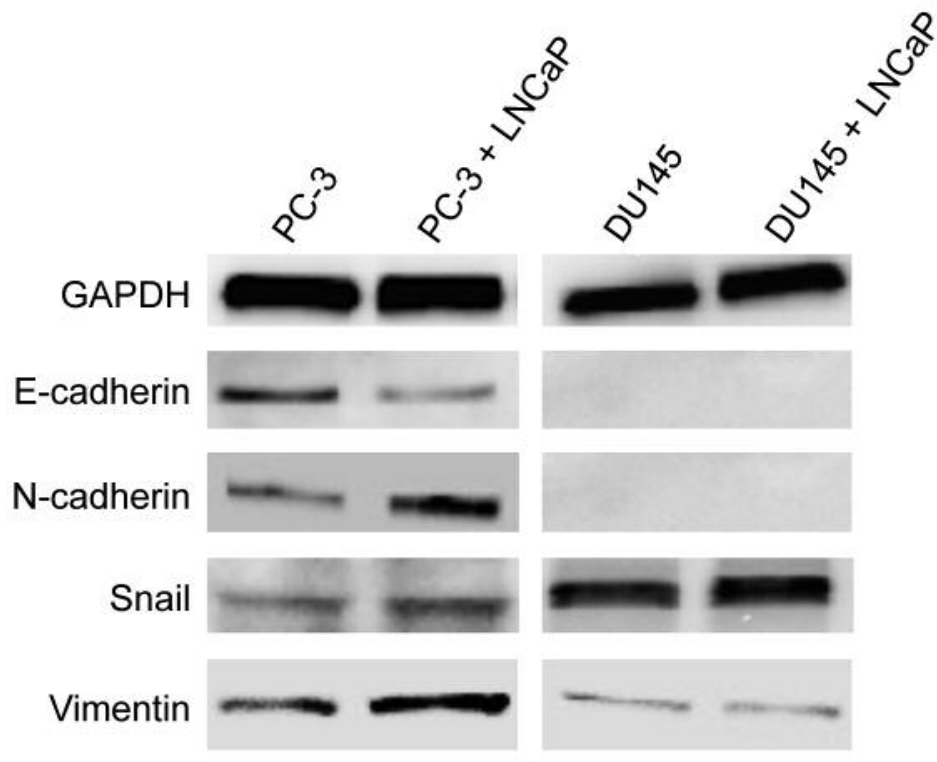

B
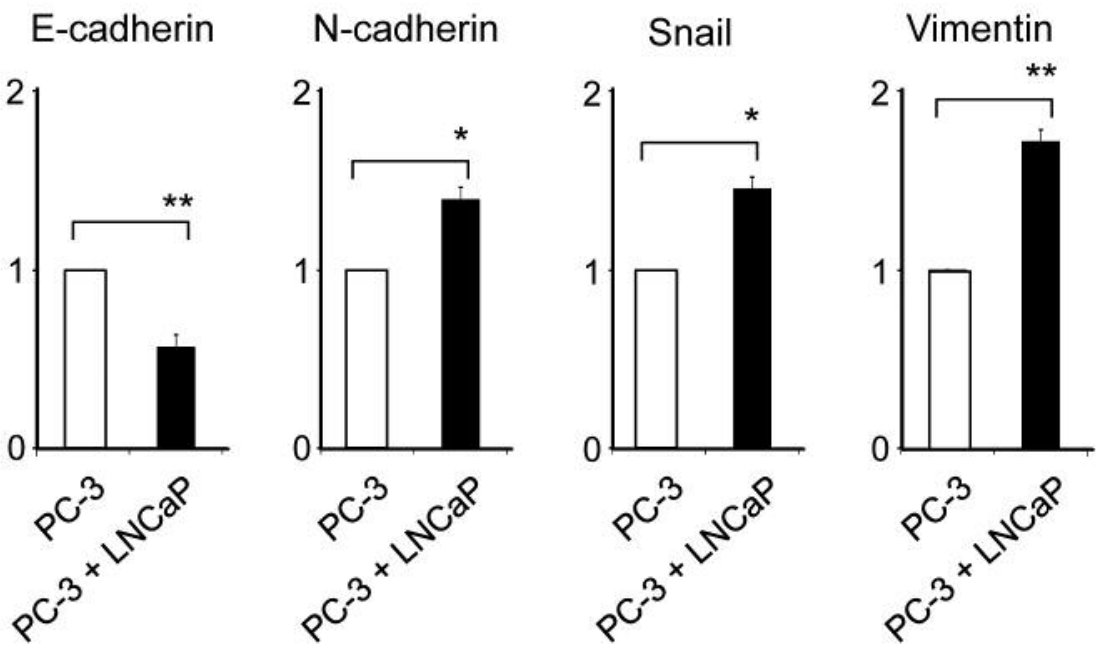

Figure 5. Western blot analysis of proteins related to epithelial-mesenchymal transition (EMT). (A) DU145 and PC-3 cells were co-cultured with LNCaP cells were plated (no contact) for $24 \mathrm{~h}$. Subsequently, protein extract from DU145 and PC-3 was analyzed for the expression of EMT-related proteins. (B) The relative intensity of each protein band for PC-3 cell monoculture and PC-3 cells co-cultured with LNCaP cells. The intensity of asterisks represents statistical significance $\left({ }^{*} p<0.05, * * p<0.01\right.$, compared to the control-monoculture).

when LNCaP cells were co-cultured with PC-3 cells, LNCaP cell proliferation was not altered, irrespective of DHT presence. It was also examined whether androgenindependent cells affect AR activity in LNCaP cells in the same model (Figure 2B). The PSA promoter activity induced by 0.1 or $1.0 \mathrm{nM}$ DHT was further elevated (2.1-fold, $p<0.001$ and 1.8 -fold $p<0.0001$, respectively) in co-culture with DU145 cells, compared to LNCaP monoculture. However, the PSA promoter activity, induced by $1.0 \mathrm{nM}$
DHT, was rather repressed by co-culture with PC-3 cells compared to LNCaP monoculture ( 0.6 -fold, $p<0.001)$.

In order to examine the effect of androgen-independent cells on the DHT metabolism in LNCaP cells, DHT concentration was measured in the medium of LNCaP cells and androgen-independent cell co-culture, $24 \mathrm{~h}$ after DHT treatment (Figure 2C). In LNCaP monoculture (24 h) $1 \mathrm{nM}$ DHT was degraded to $0.16 \mathrm{nM}$, while co-culture with PC-3 cells increased DHT concentration to $0.23 \mathrm{nM}(p<0.0001$, 
compared to monoculture control). In contrast, DHT concentration in the medium of LNCaP cells was not changed in co-culture of LNCaP with DU145 cells.

LNCaP cells enhance the proliferation of DU145 and PC-3 cells, in co-culture. We further investigated whether LNCaP cells stimulated the proliferation of DU145 and PC-3 cells. When DU145 and PC-3 cells were co-cultured with LNCaP cells, the proliferation of DU145 and PC-3 cells was stimulated by LNCaP cells (1.77-fold, $p<0.01$ and 1.43 -fold, $p<0.05$, respectively), irrespective of DHT presence (Figure $3)$. This stimulation was not inhibited by antiandrogen enzalutamide $(10 \mu \mathrm{M})$. Therefore, it was assumed that some soluble factors from $\mathrm{LNCaP}$ cells might induce the proliferation of androgen-independent cells. To test this, CM was collected from LNCaP cell culture and it was shown to stimulate the proliferation of PC-3 (at CM dilution1:4, 1.61fold, $p<0.01$; at CM dilution 1:2, 1.7-fold, $p<0.001)$ and DU145 cells (at CM dilution 1:2, 1.32-fold, $p<0.01$ ) (Figure 3B). These results indicated that soluble factors secreted by LNCaP cells cultured in vitro, could affect the proliferation of androgen-insensitive cells.

Each cell lines have an influence on each migration and invasion ability. In order to investigate how LNCaP cells affect migration and invasion of PC-3 or DU145 cells, migration of DU145 and PC-3 cells was evaluated using 2layer chamber. When DU145 cells were co-cultured with LNCaP cells for $24 \mathrm{~h}$, migration of DU145 cells was suppressed (0.21-fold, $p<0.001)$, compared with LNCaP monoculture control (Figure 4A). In contrast, when PC-3 cells were co-cultured with LNCaP cells, migration of PC-3 cells was rather promoted $(3.27$-fold, $p<0.001)$ in the presence of LNCaP cells (Figure 4B). Although migration of LNCaP cells was examined by 2-layer chamber method and wound healing assay in the presence of PC-3 or DU145, no significant difference was observed (data not shown).

Moreover, DU145 and PC-3 cell invasion was examined using 2-layer chambers with Matrigel barrier. Co-culture with LNCaP cells was shown to suppress invasion of DU145 cells (0.7-fold, $p>0.01$ ) (Figure 4C), while contrarily, promoted invasion of PC-3 cells (1.84-fold, $p>0.01$ ) (Figure 4D).

EMT was related to $P C-3$ cell migration and invasion. In order to explore whether migration and invasion of $\mathrm{PC}-3$ cells induced by LNCaP cells is associated with EMT, the expression of EMT-related proteins were examined. After coculture of PC-3 cells with LNCaP cells for $24 \mathrm{~h}$, the expression level of E-cadherin was significantly decreased (0.56-fold, $p<0.01)$, and the expression level of $\mathrm{N}$-cadherin (1.40-fold, $p<0.05)$, SNAIL (1.45-fold, $p<0.05)$, and vimentin proteins $(1.71$-fold, $p<0.05)$ was increased, but not in DU145 cells (Figure 5). These results indicated that

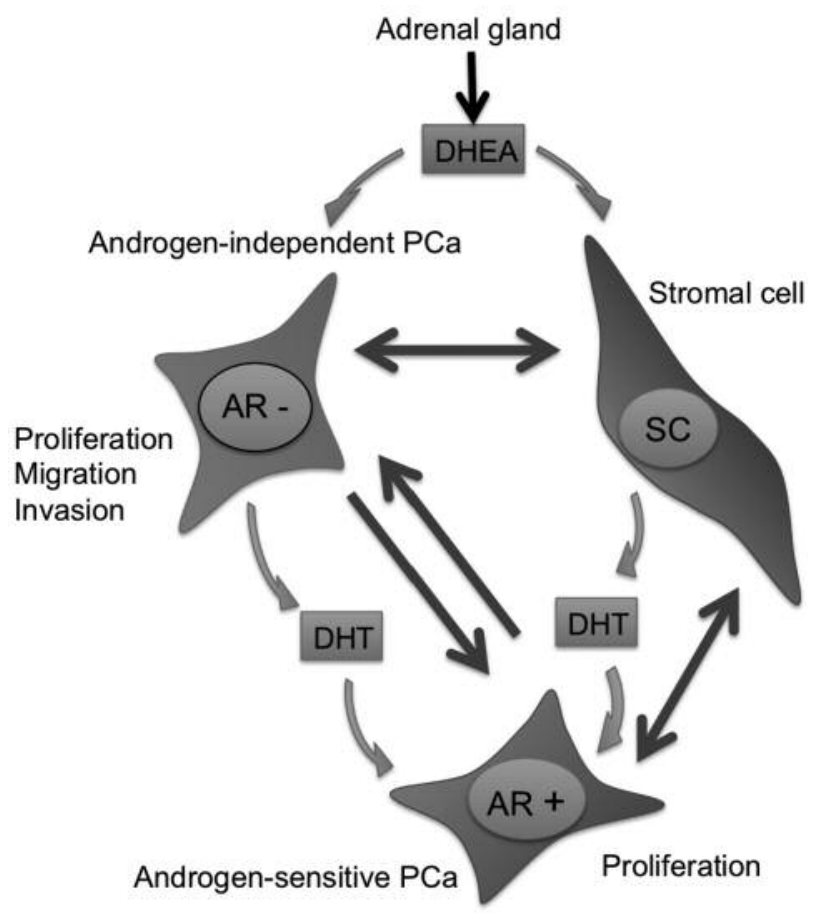

Figure 6. Crosstalk among androgen-sensitive prostate cancer (PCa) cells, androgen-independent PCa cells, and PCa-derived stromal cells (SC). Dihydrotestosterone (DHT), metabolized from dehydroepiandrosterone (DHEA) in androgen-independent PCa cells (AR negative cells, AR-) as well as in stromal cells, activates androgen receptor $(A R)$ in androgensensitive PCa cells (AR positive cells, $A R^{+}$). Crosstalk among these cells may promote the migration and invasion potential of androgenindependent PCa cells via epithelial-mesenchymal transition.

factors secreted by LNCaP cells might promote migration and invasion of PC-3 cells via EMT.

\section{Discussion}

Tumor microenvironment consists of a variety of cell types that play a pivotal role in cancer cell proliferation, and tumor progression. Especially, CAFs and bone marrow-derived myeloid cells such as tumor-associated macrophages (TAMs), tumor-associated neutrophils (TANs), and myeloid-derived suppressor cells (MDSCs), affect cancer progression, EMT, angiogenesis, and resistance to anticancer therapies (25-28). Recently, immortalized CAFs have been reported to promote proliferation and invasion of PCa cells (29). CAFs are also involved in intratumoral synthesis of androgens during the progress of PCa to CRPC (10). Since PCa tissue consists of heterogeneous cell populations, clonal selection of androgeninsensitive PCa cells is thought as one of the mechanisms by which PCa progresses to CRPC during hormonal therapy (5). However, the interaction between androgen-sensitive PCa cells and androgen-insensitive PCa cells has hardly been described. 
Our hypothesis was that, within the tumor microenvironment, androgen-sensitive $\mathrm{PCa}$ cells interact with androgen-insensitive $\mathrm{PCa}$, contributing to the progress of $\mathrm{PCa}$ to CRPC (Figure 6). In the present study, a crosstalk between androgen-sensitive LNCaP cells and androgen-independent PC-3 or DU145 cells was demonstrated. First, it was confirmed that DU145 cells, though not PC-3 cells, further enhance the proliferation rate and DHEA-induced AR activity in LNCaP cells. Furthermore, the concentration of androgens, testosterone and DHT, was shown to increase when LNCaP cells were co-cultured with DU145 cells, compared to the monoculture. We have previously reported that HSD17B5, which converts DHEA to androstenediol, was well-expressed in DU145 cells and PCa-derived stromal cells, compared to PC-3 and LNCaP cells. However, the expression level of $3 \beta$ hydroxysteroid dehydrogenase (HSD3B) that converts androstenediol to testosterone was shown to be lower in DU145 cells and PCa-derived stromal cells, compared to LNCaP cells (10). This evidence combined with the results presented here, suggest that DU145 cells as well as PCaderived stromal cells might coordinately contribute to androgen biosynthesis from DHEA to DHT and activate AR in LNCaP cells. In other words, DU145 cells and PCaderived stromal cells exhibited a similar behavior regarding androgen biosynthesis.

Moreover, DU145 cells further enhanced DHT-induced LNCaP cell proliferation and AR activity. AR has been reported to be activated in $\mathrm{PCa}$ cells by growth factor and cytokines, such as insulin-like growth factor-I (IGF-1), keratinocyte growth factor (KGF), epidermal growth factor (EGF), and interleukin 6 (IL-6) $(30,31)$. In addition, DU145 cells have been shown to secrete interleukin-6 (IL-6) (32), indicating that, apart from the enhanced expression of androgen biosynthesis-related enzymes, DU145 cells might induce AR activation via secreting cytokines. Taken together, androgen-insensitive $\mathrm{PCa}$ cells are shown to contribute to the adaption of androgen-sensitive PCa cells to the low androgen concentration, and acceleration of cell proliferation during ADT, in a similar manner as $\mathrm{PCa}-$ derived stromal cells do.

Interestingly, $\mathrm{AR}$ activity in $\mathrm{LNCaP}$ cells was reduced after co-culture with PC-3 cells, although LNCaP and PC3 cells increased the concentration of DHT in the medium (Figure $2 \mathrm{~B}$ and $2 \mathrm{C}$ ). A possible mechanism implicated in this might be the regulation of DHT efflux by Pglycoprotein, which has been reported to reduce androgen accumulation in $\mathrm{LNCaP}$ cells, thus reducing androgen responsiveness (33). The reduction of $\mathrm{AR}$ activity in LNCaP cells by co-culture with PC-3 cells should be further investigated.

Concerning the effect of LNCaP cells on androgenindependent cells, they promoted proliferation of PC-3 and DU145 cells. LNCaP cells also increased the migration and invasion of PC-3 cells, whereas they had the opposite effect on DU145 cells. It has been previously reported that monocyte chemotactic protein-1 (MCP-1) is secreted form various cells including LNCaP cells (34). MCP-1 has been shown to stimulate proliferation and increase invasion of PC3 and DU145 cells (35). These reports suggest that secreted proteins from LNCaP cells might regulate proliferation and invasion of androgen-independent PCa cells. On the other hand, migration and invasion of DU145 cells were rather suppressed by LNCaP cells. Hence, further investigation is necessary to reveal the mechanism of this interaction.

The interactions between androgen-sensitive PCa cells and androgen-independent PCa cells are critical for the selection of the appropriate treatment method. ADT is the most frequently used first-line therapy for patients with progressive PCa. However, according to the literature, ADT results in the expansion of androgen-insensitive PCa cells, which coexist with androgen-sensitive PCa cell populations in advanced $\mathrm{PCa}$, thereby promoting progress to CRPC (5). After progression to CRPC, new hormonal therapy (abiraterone acetate or enzalutamide) is often employed as an initial treatment, and docetaxel is employed as a next line treatment $(14,15)$. Interestingly, recent clinical studies revealed that chemohormonal therapy was beneficial in metastatic PCa as an initial therapy, compared with standard hormonal therapy $(36,37)$. Our results along with the evidence presented above, suggests that treatments targeted to both androgen-sensitive and -insensitive PCa cells, preventing cross-talk between the cells, should be considered before progression to CRPC.

Taken together, data from the present and our previous studies support that interplay among androgen-sensitive PCa cells, androgen-insensitive PCa cells, and PCa-derived stromal cells induces androgen synthesis in the $\mathrm{PCa}$ microenvironment (10). This interaction might contribute to the development and progression of CRPC. Therefore, it is important to disrupt the interaction among androgensensitive PCa cells, androgen-insensitive PCa cells, and PCaderived stromal cells, aiming to improve treatment efficacy.

\section{Conclusion}

Crosstalk between androgen-sensitive and androgeninsensitive PCa cells was shown to promote androgen synthesis, AR, and EMT, in vitro, and might contribute to CRPC development. Therefore, our results indicate that treatments targeted against androgen-independent PCa cells should be considered for advanced PCa therapy, in order to prevent/delay the progression of the disease.

\section{Conflicts of Interest}

The Authors declare no conflict of interest. 


\section{Acknowledgements}

This work was supported by Grants from JSPS KAKENHI (Grant Number 17H04325). We thank S. Fujinuma for skilled technical assistance (Kanazawa University).

\section{References}

1 Siegel RL, Miller KD and Jemal A: Cancer Statistics, 2017. CA: Cancer J Clin 67: 7-30, 2017.

2 Huggins $\mathrm{C}$ and Hodges CV: Studies on prostatic cancer. I. The effect of castration, of estrogen and androgen injection on serum phosphatases in metastatic carcinoma of the prostate. CA: Cancer J Clin 22: 232-240, 1972.

3 Feldman BJ and Feldman D: The development of androgenindependent prostate cancer. Nat Rev Cancer 1: 34-45, 2001.

4 Dutt SS and Gao AC: Molecular mechanisms of castrationresistant prostate cancer progression. Future Oncol 5: 1403$1413,2009$.

5 Craft N, Chhor C, Tran C, Belldegrun A, DeKernion J, Witte ON, Said J, Reiter RE and Sawyers CL: Evidence for clonal outgrowth of androgen-independent prostate cancer cells from androgen-dependent tumors through a two-step process. Cancer Res 59: 5030-5036, 1999.

6 Tombal B: What is the pathophysiology of a hormone-resistant prostate tumour? Eur J Cancer 47(Suppl 3): S179-188, 2011.

7 Labrie F, Luu-The V, Belanger A, Lin SX, Simard J, Pelletier G and Labrie C: Is dehydroepiandrosterone a hormone? J Endocrinol 187: 169-196, 2005.

8 Stanbrough M, Bubley GJ, Ross K, Golub TR, Rubin MA, Penning TM, Febbo PG and Balk SP: Increased expression of genes converting adrenal androgens to testosterone in androgenindependent prostate cancer. Cancer Res 66: 2815-2825, 2006.

9 Arnold JT, Gray NE, Jacobowitz K, Viswanathan L, Cheung PW, McFann KK, Le H and Blackman MR: Human prostate stromal cells stimulate increased PSA production in DHEA-treated prostate cancer epithelial cells. J Steroid Biochem Mol Biol 111: 240-246, 2008.

10 Mizokami A, Koh E, Izumi K, Narimoto K, Takeda M, Honma S, Dai J, Keller E and Namiki M: Prostate cancer stromal cells and LNCaP cells coordinately activate the androgen receptor through synthesis of $\mathrm{T}$ and DHT from DHEA. Endocr Relat Cancer 16: 1139-1155, 2009.

11 Ren G, Esposito $M$ and Kang Y: Bone metastasis and the metastatic niche. J Mol Med (Berl) 93: 1203-1212, 2015.

12 Centrella M, Horowitz MC, Wozney JM and McCarthy TL: Transforming growth factor-beta gene family members and bone. Endocr Rev 15: 27-39, 1994.

13 Piao YS, Wiesenfeld P, Sprando R and Arnold JT: TGFbeta1 alters androgenic metabolites and hydroxysteroid dehydrogenase enzyme expression in human prostate reactive stromal primary cells: Is steroid metabolism altered by prostate reactive stromal microenvironment? J Steroid Biochem Mol Biol 138: 206-13, 2013.

14 Beer TM, Armstrong AJ, Rathkopf DE, Loriot Y, Sternberg CN, Higano CS, Iversen P, Bhattacharya S, Carles J, Chowdhury S, Davis ID, de Bono JS, Evans CP, Fizazi K, Joshua AM, Kim CS, Kimura G, Mainwaring P, Mansbach H, Miller K, Noonberg SB, Perabo F, Phung D, Saad F, Scher HI, Taplin ME, Venner PM,
Tombal B and Investigators P: Enzalutamide in metastatic prostate cancer before chemotherapy. N Engl J Med 371: 424433, 2014.

15 Ryan CJ, Smith MR, de Bono JS, Molina A, Logothetis CJ, de Souza P, Fizazi K, Mainwaring P, Piulats JM, Ng S, Carles J, Mulders PF, Basch E, Small EJ, Saad F, Schrijvers D, Van Poppel H, Mukherjee SD, Suttmann H, Gerritsen WR, Flaig TW, George DJ, Yu EY, Efstathiou E, Pantuck A, Winquist E, Higano CS, Taplin ME, Park Y, Kheoh T, Griffin T, Scher HI, Rathkopf DE and Investigators C-A-: Abiraterone in metastatic prostate cancer without previous chemotherapy. N Engl J Med 368: 138-148, 2013.

16 Kang M, Jeong CW, Kwak C, Ku JH and Kim HH: Comparing the clinical efficacy of abiraterone acetate, enzalutamide, and orteronel in patients with metastatic castration-resistant prostate cancer by performing a network meta-analysis of eight randomized controlled trials. Oncotarget 8: 59690-59697, 2017.

17 Desmouliere A, Guyot C and Gabbiani G: The stroma reaction myofibroblast: a key player in the control of tumor cell behavior. Int J Dev Biol 48: 509-517, 2004.

18 Niu YN and Xia SJ: Stroma-epithelium crosstalk in prostate cancer. Asian J Androl 11: 28-35, 2009.

19 Giannoni E, Bianchini F, Masieri L, Serni S, Torre E, Calorini $\mathrm{L}$ and Chiarugi P: Reciprocal activation of prostate cancer cells and cancer-associated fibroblasts stimulates epithelialmesenchymal transition and cancer stemness. Cancer Res 70: 6945-6956, 2010.

20 Morrissey C and Vessella RL: The role of tumor microenvironment in prostate cancer bone metastasis. J Cell Biochem 101: 873-886, 2007.

21 Bussard KM, Gay CV and Mastro AM: The bone microenvironment in metastasis; what is special about bone? Cancer Metastasis Rev 27: 41-55, 2008.

22 Cooper CR and Pienta KJ: Cell adhesion and chemotaxis in prostate cancer metastasis to bone: a minireview. Prostate Cancer Prostatic Dis 3: 6-12, 2000.

23 Mizokami A, Gotoh A, Yamada H, Keller ET and Matsumoto T: Tumor necrosis factor-alpha represses androgen sensitivity in the LNCaP prostate cancer cell line. J Urol 164: 800-805, 2000.

24 Kim J, Mizokami A, Shin M, Izumi K, Konaka H, Kadono Y, Kitagawa Y, Keller ET, Zhang J and Namiki M: SOD3 acts as a tumor suppressor in PC-3 prostate cancer cells via hydrogen peroxide accumulation. Anticancer Res 34: 2821-2831, 2014.

25 Son B, Lee S, Youn H, Kim E, Kim W and Youn B: The role of tumor microenvironment in therapeutic resistance. Oncotarget 8 : 3933-3945, 2017.

26 Izumi K, Fang LY, Mizokami A, Namiki M, Li L, Lin WJ and Chang C: Targeting the androgen receptor with siRNA promotes prostate cancer metastasis through enhanced macrophage recruitment via CCL2/CCR2-induced STAT3 activation. EMBO Mol Med 5: 1383-1401, 2013.

27 Josson S, Matsuoka Y, Chung LW, Zhau HE and Wang R: Tumor-stroma co-evolution in prostate cancer progression and metastasis. Semin Cell Dev Biol 21: 26-32, 2010.

28 Murdoch C, Muthana M, Coffelt SB and Lewis CE: The role of myeloid cells in the promotion of tumour angiogenesis. Nat Rev Cancer 8: 618-631, 2008.

29 Yu S, Jiang Y, Wan F, Wu J, Gao Z and Liu D: Immortalized cancer-associated fibroblasts promote prostate cancer carcinogenesis, proliferation and invasion. Anticancer Res 37: 4311-4318, 2017. 
30 Culig Z, Hobisch A, Cronauer MV, Radmayr C, Trapman J, Hittmair A, Bartsch $\mathrm{G}$ and Klocker H: Androgen receptor activation in prostatic tumor cell lines by insulin-like growth factor-I, keratinocyte growth factor, and epidermal growth factor. Cancer Res 54: 5474-5478, 1994.

31 Matsuda T, Junicho A, Yamamoto T, Kishi H, Korkmaz K, Saatcioglu F, Fuse H and Muraguchi A: Cross-talk between signal transducer and activator of transcription 3 and androgen receptor signaling in prostate carcinoma cells. Biochem Biophys Res Commun 283: 179-187, 2001.

32 Inokuchi J, Narula N, Yee DS, Skarecky DW, Lau A, Ornstein DK and Tyson DR: Annexin A2 positively contributes to the malignant phenotype and secretion of IL-6 in DU145 prostate cancer cells. Int J cancer 124: 68-74, 2009.

33 Fedoruk MN, Gimenez-Bonafe P, Guns ES, Mayer LD and Nelson CC: P-glycoprotein increases the efflux of the androgen dihydrotestosterone and reduces androgen responsive gene activity in prostate tumor cells. Prostate 59: 77-90, 2004.

$34 \mathrm{Lu}$ Y, Cai Z, Galson DL, Xiao G, Liu Y, George DE, Melhem MF, Yao Z and Zhang J: Monocyte chemotactic protein-1 (MCP1) acts as a paracrine and autocrine factor for prostate cancer growth and invasion. Prostate 66: 1311-1318, 2006.

35 Ito Y, Ishiguro H, Kobayashi N, Hasumi H, Watanabe M, Yao M and Uemura $\mathrm{H}$ : Adipocyte-derived monocyte chemotactic protein-1 (MCP-1) promotes prostate cancer progression through the induction of MMP-2 activity. Prostate 75: 1009-1019, 2015.
36 Sweeney CJ, Chen YH, Carducci M, Liu G, Jarrard DF, Eisenberger M, Wong YN, Hahn N, Kohli M, Cooney MM, Dreicer R, Vogelzang NJ, Picus J, Shevrin D, Hussain M, Garcia JA and DiPaola RS: Chemohormonal therapy in metastatic hormone-sensitive prostate cancer. N Engl J Med 373: 737-746, 2015.

37 James ND, Sydes MR, Clarke NW, Mason MD, Dearnaley DP, Spears MR, Ritchie AW, Parker CC, Russell JM, Attard G, de Bono J, Cross W, Jones RJ, Thalmann G, Amos C, Matheson D, Millman R, Alzouebi M, Beesley S, Birtle AJ, Brock S, Cathomas R, Chakraborti P, Chowdhury S, Cook A, Elliott T, Gale J, Gibbs S, Graham JD, Hetherington J, Hughes R, Laing R, McKinna F, McLaren DB, O'Sullivan JM, Parikh O, Peedell C, Protheroe A, Robinson AJ, Srihari N, Srinivasan R, Staffurth J, Sundar S, Tolan S, Tsang D, Wagstaff J, Parmar MK and investigators S: Addition of docetaxel, zoledronic acid, or both to first-line long-term hormone therapy in prostate cancer (STAMPEDE): survival results from an adaptive, multiarm, multistage, platform randomised controlled trial. Lancet 387: 1163-1177, 2016.

Received November 13, 2017

Revised February 14, 2018

Accepted February 26, 2018 